\title{
Availability comparison of two all-optical network approaches
}

\author{
BRANKO MIKAC, ROBERT INKRET \\ University of Zagreb, Croatia
}

\begin{abstract}
Two all-optical network approaches, the partitioned and grid connect, are compared from the availability point of view. The availability measures are defined. The availability model and calculation procedure are described. The comparison of availability performances has been done for an example network. The availability figures are analyzed and proposals for availability design improvement are given.
\end{abstract}

Keywords: availability, all-optical network

\section{INTRODUCTION}

The paper presents the results of an availability performance analysis which has been carried out within the European project COST 239 - "Ultra-high Capacity Optical Transmission Networks", aim of which is to study feasibility of an European all-optical network (EON), capable of carrying all the international traffic between the European main centers. Different studies of the all-optical network design resulted in two approaches to be evaluated. The main differences of the approaches are in overcoming the problem of both of transmission distance limitation and of topology regularity.

The partitioned network approach provides more optical domains assigned to subnetworks. The core network is highly meshed topology with high percentage of all network traffic. Each peripheral ring sub-network is connected to the core through opto-electronic interfaces (Figure 1). Within the optical domain a re-configurable optical path layer could be established. The topology is optimized according to the cost criteria, fulfilling traffic and fault tolerance requirements, providing the shortest paths between all pairs of nodes. 

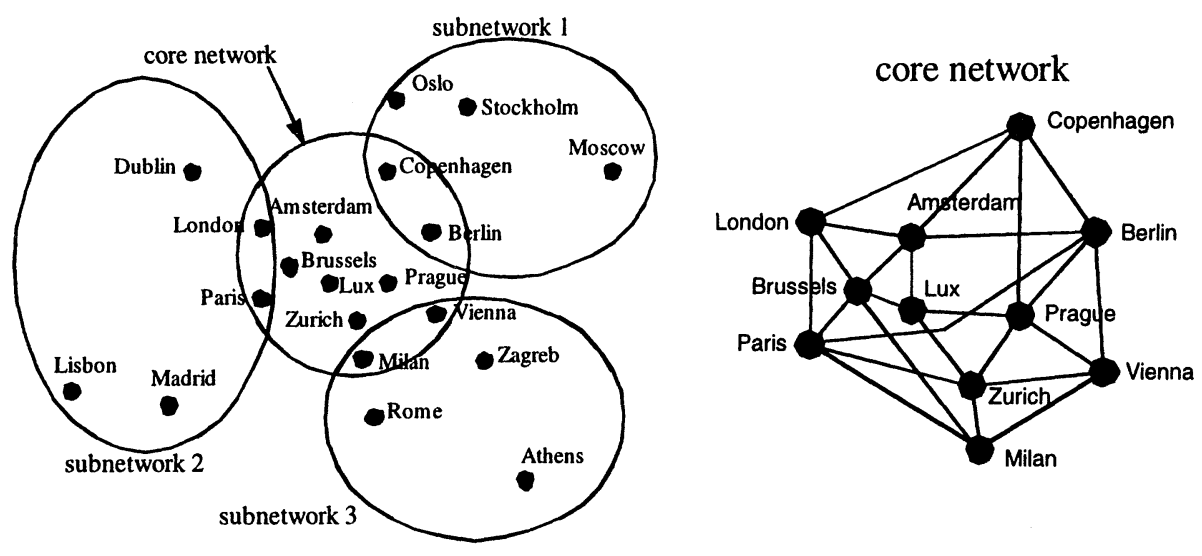

Fig. 1- Partitioning network - COST 239 case study

In the grid connect network approach a regular topology is used as a virtual one (Figure 2) on the multiplex section layer. In order to maintain the regularity of the network, fulfilling asymetric traffic requirements, "twinnodes" were introduced (white circles in figure 2). The regularity results in a uniform node structure of low complexity. In this approach the wavelength routing is static. There is no re-configurability in the optical domain (fixed path layer). The optical domain is limited to the predefined depth. In this paper the depth of 2 is assumed comprising for each node an optical domain with 24 surounded neighborous.

In the paper both of approaches have to be compared in terms of availability, analyzing an example sub-network, designed to provide comparable results.

\section{ASSUMPTIONS}

All the availability measures to be calculated are related to node pair availability ( $s$ $t$ availability). Steady-state (asymptotic) availabilities are considered, assuming constant failure and repair rates. The failures in the network are caused by non-self healing outages; every failure has to be repaired. The impact of the node unavailabilities is negligible as compared with the availabilities of optical links. Link failures are assumed to be caused by a failures in the fiber optical amplifiers, or in the fiber cables, causing an interruption of all services in the cable (see the Fig.3). This 
assumption is introduced because the data from the field show that the most of failures are caused by "outside interference", dig-ups, craft/workman errors, etc., affecting a high percentage of fibers in the cable.
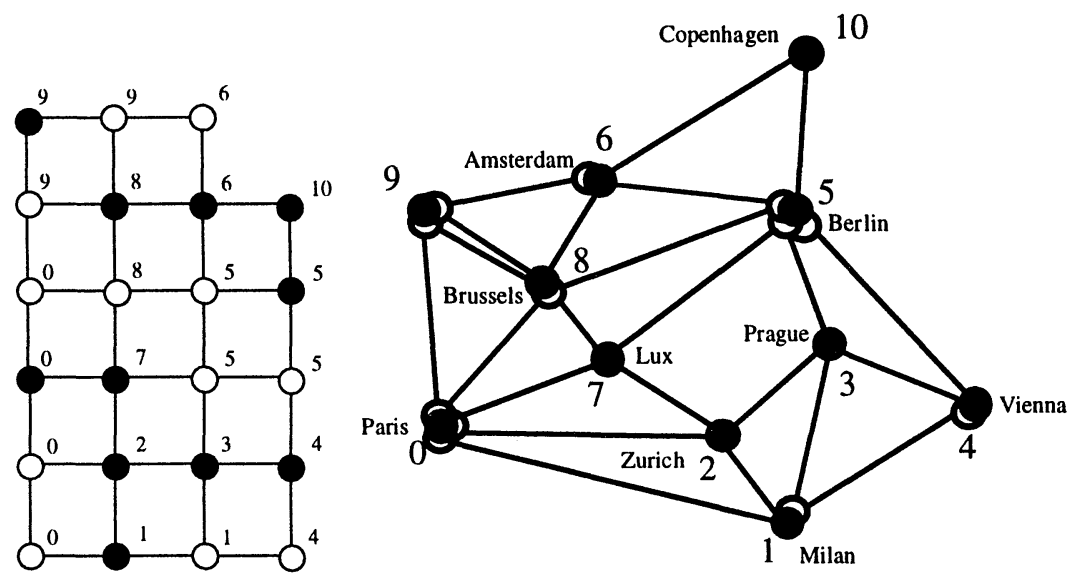

Fig. 2 - The WDM-Gridconnect - the COST 239 case study

Availability of one path element is taken to be $A \approx \lambda \cdot M T T R$ where $\lambda$ is failure rate and MTTR is mean time to repair. The values for the $\lambda$ and MTTR are assumed to be: $\lambda_{\mathrm{POA}}=\lambda_{\mathrm{BOA}}=2000$ fit (1fit=number of failures per $10^{9}$ hours), $M T T R_{\mathrm{POA}}=M T T R_{\mathrm{BOA}}=6 \mathrm{~h}, \lambda_{\text {cable }}=114 \mathrm{fit} / \mathrm{km}, \lambda_{\mathrm{OA}}=4500 \mathrm{fit}, M T T R_{\text {cable }}=M T T R_{\mathrm{OA}}=21$ $\mathrm{h}$. The failures of optical cables and optical amplifiers are mutually independent. The number of optical amplifiers is directly related to the distance between the optical amplifiers. The distance between optical amplifiers is assumed to be $100 \mathrm{~km}$. 


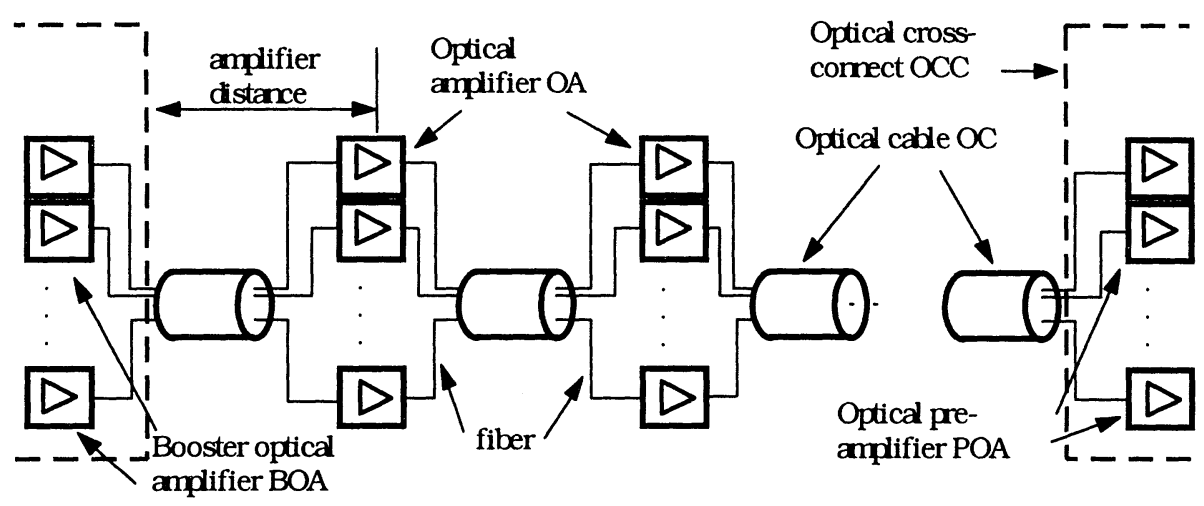

Fig. 3 - Optical link

\section{AVAILABILITY MODEL}

In order to compare the all-optical network approaches from the availability point of view, node pair connection terms were defined and the corresponding availability measures have been adopted. Every term has double meaning: it describes the type of the network connection, and on the other hand, it denotes a logical variable describing the faulty-free condition of the connection, if the variable is in the noncomplementary state. The probability of non-complementary state is equal to availability performance. In complementary state logical variable denotes faulty condition. The probability of complementary state is equal to unavailability performance. Which expression will be chosen depends on the expression complexity, given by the number of product terms.

The logical connection $L C_{i j}$ is a bi-directional connection between nodes $i$ and $j$ which fulfills all the traffic or capacity requirements in the directions $(i, j)$ and $(j, i)$. (As a logical variable $L C_{i j}$ denotes a faulty-free condition of logical connection.)

The logical connection between nodes $i$ and $j$ is composed of one or more logical channels.

The logical channel $K_{i j}$ is a bi-directional connection between the nodes $i$ and $j$ which has an indivisible capacity in the optical domain, corresponding in WDM to one wavelength channel at standard bit rates $(2.5 \mathrm{Gbit} / \mathrm{s}, 10 \mathrm{Gbit} / \mathrm{s}$, etc.) for each path.

The relationship between the logical connection $L C$ and the logical channels $K(q)$, $q=1,2, \ldots, n$ could be defined, in general, by the $k$-out-of- $n$ principle. Let us consider two cases. 
In the case where $k=n$, one can assume that the logical connection contain no spare capacity. In order to fulfill the traffic requirements, all of $n$ logical channels should be in a faulty-free condition, and the successful event of logical connection could be described by Eq. (1'). The complementary form $\overline{L C}(n)$, denoted by $\left(1^{\prime \prime}\right)$ is given too:

$$
L C(n)=\bigcap_{q=1}^{n} K(q), \quad \overline{L C}(n)=\bigcup_{q=1}^{n} \bar{K}(q)
$$

where $\bigcap$ is logical AND, and $\bigcup$ logical OR operator.

The logical connection availability of type $A[L C(n)]$ and unavailability of type $U[\overline{L C}(n)]$ between nodes $i$ and $j$ are defined according to the availability model in Figure 4:

$$
\begin{aligned}
& A[L C(n)]=\operatorname{Pr}\{L C(n)\}=\operatorname{Pr}\{K(1) \cap K(2) \cap \cdots \cap K(n)\} \\
& U[\overline{L C}(n)]=\operatorname{Pr}\{\overline{L C}(n)\}=\operatorname{Pr}\{\bar{K}(1) \cup \bar{K}(2) \cup \cdots \cup \bar{K}(n)\}
\end{aligned}
$$

In the case where $k<n$, the assumption is that a logical connection has enough spare capacity, fulfilling traffic requirements with at least $k$ logical channels out of $n$ (total number of channels). This case could happen, if, for example, the electrical SDH level provides a spare capacity for protection purposes. In this case the faulty(free) condition of logical connection is:

$$
L C(k, n)=\bigcup_{p=1}^{M}\left[\bigcap_{r \in C p}^{k} K(r)\right], \quad \overline{L C}(k, n)=\bigcap_{p=1}^{M}\left[\bigcup_{r \in C p}^{k} \bar{K}(r)\right], \quad M=\left(\begin{array}{l}
n \\
k
\end{array}\right)
$$

where $K(r),(\bar{K}(r))$ is $r$-th element (logical channel) out of $k$, in combination $C_{p}$.

The logical connection availability k-out-of-n $A[L C(k, n)]$ between two nodes is defined by:

$$
A[L C(k, n)]=\operatorname{Pr}\{L C(k, n)\}
$$


For example, the availability 2-out-of-3 $A[L C(2,3)]$ and the complementary unavailability $U[\overline{L C}(2,3)]$ for logical connection composed of 3 logical channels, are equal to:

$$
\begin{aligned}
& A[L C(2,3)]=\operatorname{Pr}\{[K(1) \cap K(2)] \cup[K(1) \cap K(3)] \cup[K(2) \cap K(3)]\} \\
& U[\overline{L C}(2,3)]=\operatorname{Pr}\{[\bar{K}(1) \cup \bar{K}(2)] \cap[\bar{K}(1) \cup \bar{K}(3)] \cap[\bar{K}(2) \cup \bar{K}(3)]\}\left(5^{\prime \prime}\right)
\end{aligned}
$$

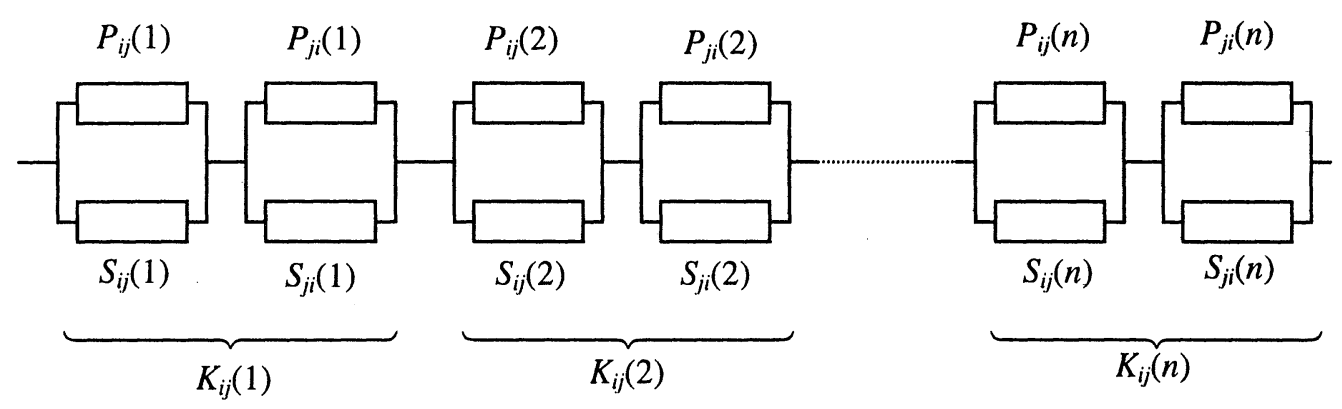

Fig. 4 - Availability model of logical connection for $k=n$

In the topologies under consideration the logical channel is composed of two paths, in redundant structure. In the partitioned network the primary path is used in a faulty-free state of the network, and its spare path is used if the primary path is faulty. In the grid-connect network both paths, primary and spare, are used in parallel at the same time, as hot stand-by redundancy.

According to the model (Fig.4), the logical channel $K_{i j}(q)$, is faulty-free, if the primary path $P_{i j}(q)$ or the spare independent path $S_{i j}(q)$ in one direction and the primary path $P_{j i}(q)$ or the spare independent path $S_{j i}(q)$ in opposite direction are faulty-free. 


$$
\begin{aligned}
& K_{i j}(q)=\left[P_{i j}(q) \cup S_{i j}(q)\right] \cap\left[P_{j i}(q) \cup S_{j i}(q)\right]= \\
& =\left[P_{i j}(q) \cap P_{j i}(q)\right] \cup\left[P_{i j}(q) \cap S_{j i}(q)\right] \cup\left[S_{i j}(q) \cap P_{j i}(q)\right] \cup\left[S_{i j}(q) \cap S_{j i}(q)\right] \\
& \quad \bar{K}_{i j}(q)=\left[\bar{P}_{i j}(q) \cap \bar{S}_{i j}(q)\right] \cup\left[\bar{P}_{j i}(q) \cap \bar{S}_{j i}(q)\right]
\end{aligned}
$$

The (un)availability of logical channel $K_{i j}(q)$ is as follows

$$
A\left[K_{i j}(q)\right]=\operatorname{Pr}\left\{K_{i j}(q)\right\} \quad U\left[K_{i j}(q)\right]=\operatorname{Pr}\left\{\bar{K}_{i j}(q)\right\}
$$

The path between the two nodes $i$ and $j$, is a chain of links $(i, x),(x, y), \ldots,(z, j)$ and nodes $(i, x, y, \ldots, z, j)$. Each link the path is passing through, is specified by an optical cable $O C_{x y}$, and a fiber $v$ in the cable with the corresponding chain of optical amplifiers $O A_{x y}(v)$. Each path is defined in WDM also by a wavelength (or more wavelengths in the case of wavelength conversion), but the wavelength assignment within a fiber has no influence on availability calculation, because all wavelengths are assumed to be equal. Node elements the path is passing through, are multiplexers (WDM), optical switches, etc. The successful event of a path, primary $P_{i j}$ or a spare path $S_{i j}$, could be expressed by (8):

$$
\text { path }_{i j}=\bigcap \text { (logical variables of path elements). }
$$

The faulty-free and faulty conditions of a path $q$ can be expressed by

$$
\operatorname{path}_{i j}(q)=\left[O C_{i x} \cap O A_{i x}(u)\right] \cap\left[O C_{x y} \cap O A_{x y}(v)\right] \cap \cdots \cap\left[O C_{z j} \cap O A_{z j}(w)\right]
$$

$$
\overline{p a t h}_{i j}(q)=\left[\overline{O C}_{i x} \cup \overline{O A}_{i x}(u)\right] \cup\left[\overline{O C}_{x y} \cup \overline{O A}_{x y}(v)\right] \cup \cdots \cup\left[\overline{O C}_{z j} \cup \overline{O A}_{z j}(w)\right]
$$

In the analysis the assumption is that the chain $O A_{x y}(v)$ is faulty-free if all amplifiers in the chain are faulty-free. (In general, any redundant structure of optical amplifiers could be modeled, as well.) 
Substituting the sets of simple events, forming expressions (9), into more complex one's, (6), (3), or (2), one can achieve (un)availability expressions for selected (un)availability measures. Table 1 shows the numbers of product terms in union.

The probability calculation to be described in Part 3 must take into account the fact that Boolean products describing different paths in the network could have many elements in common. The grade of dependency of network elements has to influence the availability figures for specific availability calculation cases.

Table 1

\begin{tabular}{|l|c|c|c|c|}
\hline & $L C(n)$ & $\overline{L C}(n)$ & $L C(k, n)$ & $\overline{L C}(k, n)$ \\
\hline $\begin{array}{l}\text { Number of } \\
\text { product terms }\end{array}$ & $4^{n}$ & $8 n l^{2}$ & $\left(\begin{array}{l}n \\
k\end{array}\right) 4^{k}$ & $\left(\begin{array}{l}n \\
k\end{array}\right) 8 n l^{2}$ \\
\hline
\end{tabular}

$n$ - number of logical channels,

$k$ - $\quad$ parameter in $k$-out-of- $n$ structure,

$l$ - average number of links in paths contributing logical channels of one logical connection.

Regarding the unavailability of logical connection $U\left(L C_{i j}\right)$, a lower $U_{L B}$ and upper bound $U_{U B}$ values for each approach can be determined by using Eq. ( $\left.2^{\prime}\right)$ :

$$
\begin{aligned}
& U\left(L C_{i j}\right) \geq U_{L B}=\max _{m}\left\{U\left(K_{i j}(q)\right)\right\} \\
& U\left(L C_{i j}\right) \leq U_{U P} \approx \sum_{m=1}^{n} U\left(K_{i j}(q)\right)
\end{aligned}
$$

The Eq. 10 shows that a minimum unavailability in the non-redundant structure of logical channels $(k=n)$ could be achieved if all logical channels of a logical connection are assumed to use for every link in the path, the same optical fiber (with different wavelengths) in one direction. This is the maximum dependency which can be achieved in the partitioned network by using minimum number of path elements. In the grid network, where different path routes exist, in non-redundant case, the unavailability can not be better than the worst channel unavailability.

The upper bound unavailability according to the Eq. 11 reflects the case of maximum independence of channels in each of analyzed approaches. The logical con- 
nection makes use of a maximum number of path elements; the channels are assigned to different optical fibers within the same cable in the partitioned network, or they are assigned to different cables in the grid network.

\section{AVAILABILITY CALCULATION}

The availability calculation to be done is based on development, minimization and transformation of a Boolean product union, until a sum of disjoint union of products is achieved, assuming each product is composed of independent events. After finishing this procedure the (un)availability performance can be calculated as a sum of Boolean product probabilities.

The (un)availability measure of the logical connection, being the most complex one, is calculated through the following steps:

1. Formation of a logical expression of the logical connection faulty-(free) condition, comprising expressions of logical channels, paths and path elements (OCs and OAs).

2. Transformation of a logical expression into the union of (non-disjoint) terms by performing AND (OR) operation on expressions (6) for the logical channel itself and adding step-by-step new terms defined by the expressions (2) or (3).

In order to reduce the exponential growth of the term number in the union, the minimization could be applied after each multiplication, according to the following rules:

$a b \cap a c=a b c, \quad a \cup a b=a \quad$ where $a, b, c$ could be any Boolean product.

3. Obtaining a disjoint union of products using the algorithm of Abraham [4].

4. Alternating the calculation of partial availability $A_{p}$ and partial unavailability $U_{p}$, as sums of term probabilities. A satisfactory result is achieved if $A_{p}-U_{p}<$ prescribed error.

\section{EXAMPLE SUBNETWORK}

In order to compare two network approaches from the availability point of view two nodes are selected (Milan, Berlin) forming an example sub-network (Figure 5) with specified logical channels, paths and fibers in both approaches. Both logical connections contain 4 logical channels within optical domain. 


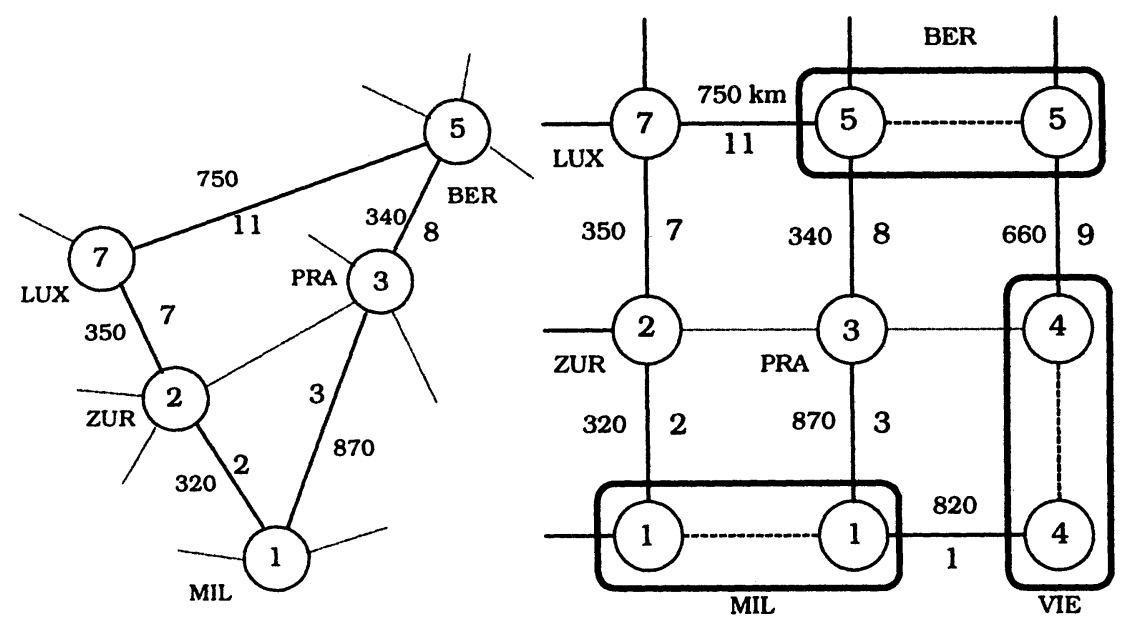

Fig. 5 - An example sub-network

In the partitioned network the paths (primary and spare) are chosen as the first and the second shortest paths, respectively. All logical channels have the same path routes. The option in the partitioned network could be the usage of fibers in optical cables. Two extreme cases will be analyzed. In the first case (P1) all paths, assigned to the same route, use only a single fiber within one optical cable, but different wavelengths. In the second case (P4) each path of all logical channels uses a separate fiber in the same optical cable. In this case for every direction $4+4$ fibers are used.

In the grid network (G) the paths and the related logical channels are chosen according to prescribed rules of regular topology. As a consequence assignment options are not possible. Different logical channels could have different path routes. At least one of logical channels in the grid network has the paths with the same routes as the logical channels in the partitioned network. The analysis should point out how this "lack of freedom" in regular network could influence on the availability performance.

The routes for the above three cases are shown in Figure 6. The logical connection is described as follows:

source_node - termination_node ( number_of_logical_channels ) \{ logical_channels \} 
For each logical channel both directions, $(>$ and $<$ ) could be defined, and for each direction a primary and a spare path are specified:

$$
\begin{array}{r}
\text { number_of_logical_channel } \\
\text { > primary_path I spare_path ] } \\
\text { < primary_path I spare_path ]; }
\end{array}
$$

The paths are described as a list of optical links $L x(f, w)$ where $L x$ denotes link $x$, and $f$ and $w$ are the assigned fiber and wavelength, respectively.

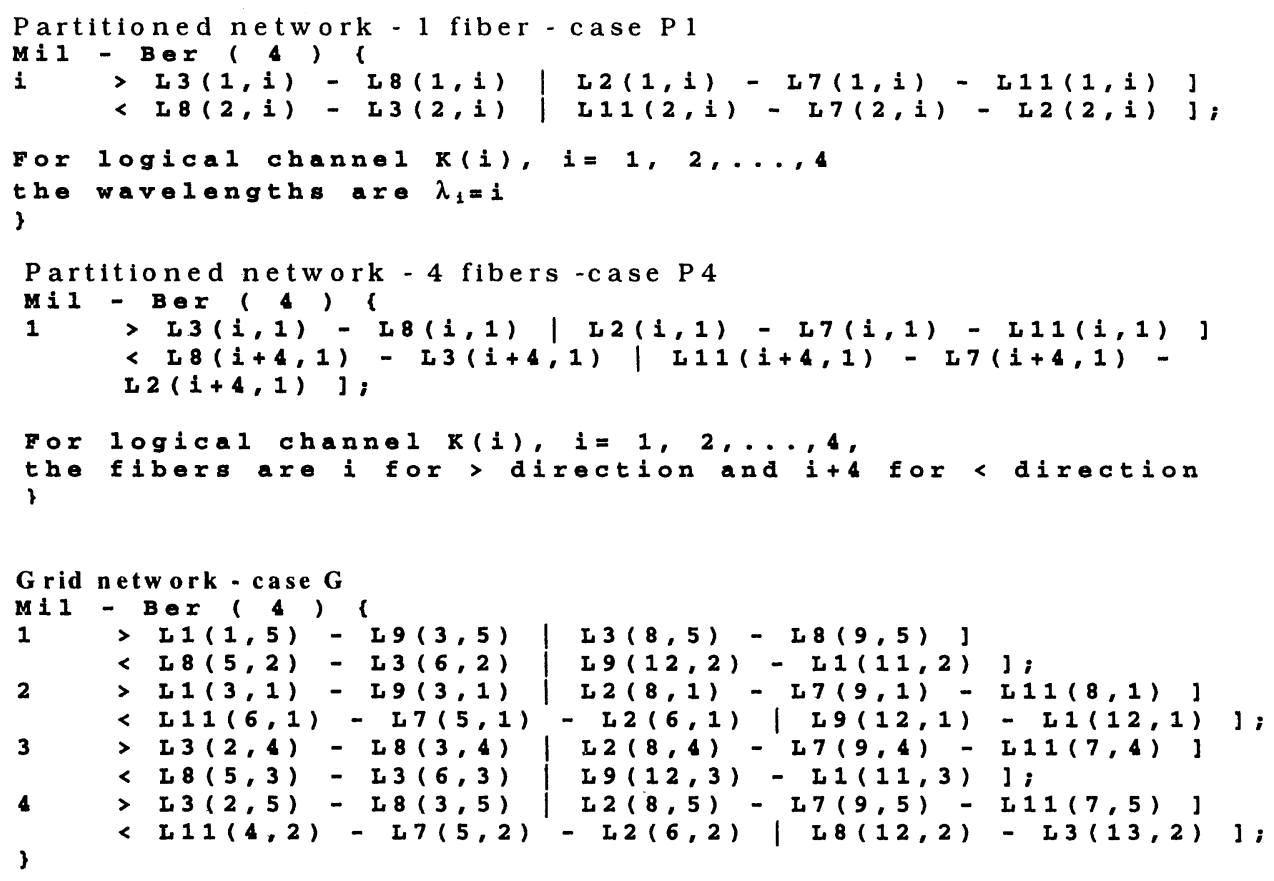

Fig. 6 - Logical connection - logical channel - path - link - fiber - wavelength assignment 


\section{AVAILABILITY FIGURES}

The results of the availability calculation for three cases, in the non-redundant and redundant structures of logical channels forming a logical connection, are shown in Table 2.

Table 2 - Availability of logical connection $\left(L C_{15}\right)$ Milan - Berlin

\begin{tabular}{|c|c|c|c|c|}
\hline & & \multicolumn{2}{|c|}{ Partitioned network } & \multirow{2}{*}{$\begin{array}{c}\text { Grid } \\
\text { network } \\
\text { Case } G\end{array}$} \\
\hline & & $\begin{array}{c}\text { Case } \\
P 1 \\
\text { (1 fiber) } \\
\end{array}$ & $\begin{array}{l}\text { Case } P 4 \\
\text { (4 fibers) }\end{array}$ & \\
\hline No. & \multicolumn{4}{|c|}{ non-redundant structure of logical channels } \\
\hline \multirow[t]{4}{*}{1} & $\begin{array}{l}\text { Unavailability of logical } \\
\text { connection } U(L C) \quad \mathbf{x} 10^{-5}\end{array}$ & 2.74914 & 8.02586 & 8.99495 \\
\hline & $\begin{array}{l}\text { No. of logical channels (both di- } \\
\text { rections) }\end{array}$ & $4+4$ & $4+4$ & $4+4$ \\
\hline & No. of fiber per $L C$ & 2 & 8 & 12 \\
\hline & \multicolumn{4}{|c|}{ Unavailability of logical channels $U(K(q)) \quad \mathbf{x 1 0}^{-5}$} \\
\hline 2 & $q=1$ & 2.74914 & 2.74914 & 3.81166 \\
\hline 3 & 2 & & & 4.16115 \\
\hline 4 & 3 & & & 2.74914 \\
\hline 5 & 4 & & & 2.74914 \\
\hline 6 & $\begin{array}{l}U_{L B}-\text { lower bound of } U(L C) \\
\max _{\mathrm{i}}\{U(K(q))\}\end{array}$ & 2.74914 & 2.74914 & 4.16115 \\
\hline \multirow[t]{3}{*}{7} & $\begin{array}{l}U_{U P}-\text { upper bound of } U(L C) \\
\sum U(K(q))\end{array}$ & 10.9966 & 10.9966 & 13.4711 \\
\hline & \multicolumn{4}{|c|}{$\begin{array}{c}\text { redundant structure of logical channels } \\
\end{array}$} \\
\hline & \multicolumn{4}{|c|}{$\begin{array}{l}\text { Unavailability of the logical connection } k \text {-out-of- } n U(L C(k, n)) \\
\text { U }\end{array}$} \\
\hline 8 & $U(L C(1,4))$ & 2.74914 & 0.98318 & 0.00611 \\
\hline 9 & $U(L C(2,4))$ & 2.74914 & 0.98314 & 1.87571 \\
\hline 10 & $U(L C(3,4))$ & 2.74914 & 1.00438 & 3.81765 \\
\hline
\end{tabular}

The comparison of the unavailabilities of the logical connection (No.1: P1 c. P4) (c. stands for compared to) for non-redundant structure shows the influence of the dependency by using a single or more fibers in the same cable. 
The comparison (No.1: $P$ c. $G$ ) gives for cases $P 1$ and $P 2$ better (lower) unavailabilities, because logical channels in grid network use more than one path route.

All logical channels (No.2 - 5: $P$ ) have the same values of unavailabilities. This is due to the fact that all channels use the same path routes (the same optical cables and equal number of optical amplifiers). The unavailabilities (No.2 - 5: $G$ ) differ because these channels use different path routes (lengths) which are not "shortest paths optimized". The more elements are used in the non-redundant structure, the higher unavailability values are achieved.

The different bound values (No.6-7: $P$ c. $G$ ) reflect the way of path-fiberwavelength assignment in the different approaches.

Comparing figures for redundant structure, the grid network shows better figures for high redundancy (No.8: $G$ c. $P$ ) and (No.9: $G$ c. $P 1)$. The more elements are used in redundant structure, the lower unavailability values are achieved.

\section{CONCLUSIONS}

In the paper the availability performances and availability model of an all-optical network are defined, and the availability calculation procedure described. The implemented tools are used to calculate and compare availability figures of two network approaches. The point was not to achieve the absolute availability figures for the selected approaches, because the availability data for the future all-optical network can not be estimated precisely enough. The comparison results intent to point out some suggestions for availability design improvement.

In the case where a non-redundant structure of logical connection is used, or for dimensioning of logical channels in any case, one should prefer the solutions where is as less number of network elements as possible. For example, a failure of an optical amplifier affects less number of logical connections, if more logical channels of one logical connection use the same fiber (the same chain of optical amplifiers). Those solutions should be preferred too, which use the same cable for logical channel path routes in both directions. The same conclusion could be stated for the usage of cross-connect elements in a node, the logical channels are passing through. Note that every design should maintain the independence of the primary and the spare paths.

If a redundant structure of the logical connection is used, one should evaluate for particular design which one of two opposite influences could overrule: the influence of the unavailabilities of equivalent logical channels, "shortest path minimized", or the influence of the unavailabilities of redundant structure comprising logical channels with different path routes, where all paths can not be the shortest one's. 


\section{REFERENCES}

[1] Tillerot F., D. R. Hjelme, "WDM survivable network requirements against transmission limitations in the pan European core network", Electron. Lett., vol. 32, pp 58-59, 1996.

[2] Jager, H.A., "The WDM-Gridconnect as a Transport Structure", IEEE Photon. Tech. Lett., vol. 7, no. 5, pp. 576-578, May 1995.

[3] Mahony, M.J., M.S. Sinclair, B. Mikac, "Ultra-High Capacity Optical Transmission Network: European Research Project, ITA, vol. 12, pp. 33-45, 1993.

[4] Abraham, J.A., "An Improved Algorithm for Network Reliability", IEEE Trans. Reliability, vol. R-28, pp. 58-61, April 1979. 